Frequency response of a focused SAW device based on concentric wave surfaces: simulation and experiment

This article has been downloaded from IOPscience. Please scroll down to see the full text article.

2005 J. Phys. D: Appl. Phys. 382986

(http://iopscience.iop.org/0022-3727/38/16/035)

The Table of Contents and more related content is available

Download details:

IP Address: 140.112.113.225

The article was downloaded on 23/12/2008 at 02:12

Please note that terms and conditions apply. 


\title{
Frequency response of a focused SAW device based on concentric wave surfaces: simulation and experiment
}

\author{
Tsung-Tsong Wu, He-Tai Tang and Yung-Yu Chen ${ }^{1}$ \\ Institute of Applied Mechanics, National Taiwan University, Taipei, Taiwan \\ E-mail: yychen@ndt.iam.ntu.edu.tw
}

Received 29 December 2004, in final form 25 February 2005

Published 5 August 2005

Online at stacks.iop.org/JPhysD/38/2986

\begin{abstract}
Focused interdigital transducers (FIDTs) based on concentric wave surfaces can excite surface acoustic wave (SAW) with high intensity, high beamwidth compression ratio and small localized area. In this paper, we developed a model to analyse the frequency response of a SAW device with FIDTs based on concentric wave surfaces. First, we constructed a focused SAW device by adopting a pair of FIDTs shaped as the concentric wave surface. To calculate its frequency response, a simulation model was established based on the 2D SAW filter model, effective permittivity approach and perturbation theory. Further, the focused SAW devices were designed and fabricated via the microelectromechanical system process technique.

A good agreement between the simulated and measured results was found. Finally, the frequency responses of focused SAW devices based on concentric wave surfaces were discussed and some attractive features were found, such as less ripples of pass-band and high stop-band rejection. In addition, in comparison with the conventional SAW devices with uniform interdigital transducers, the focused SAW devices are more sensitive to variations in the focal area, instead of the whole delayline region. Accordingly, they are suitable to be applied to detect or manipulate some localized variations, such as acousto-optic or acousto-electric effects.
\end{abstract}

(Some figures in this article are in colour only in the electronic version)

\section{Introduction}

Surface acoustic wave (SAW) devices have many useful applications, such as filters, sensors, detectors, actuators, etc. In most of the applications, realizing the frequency responses of SAW devices is very important. Although focused interdigital transducers (FIDTs) have been studied for many years, people have been concerned only with their attractive characteristics, such as high power intensity, large compression ratio and so on $[1,2]$. The investigation of the frequency response of a SAW device with FIDTs is still awaited. For focused SAW devices, the second order effects of SAW propagation, including SAW diffraction, refraction and beam

1 Author to whom any correspondence should be addressed. steering, are much stronger than those of the conventional SAW devices and deeply affect the frequency response. Therefore, to calculate accurately the frequency response of a focused SAW device, a simulation model that considers the second order effects is needed.

The main purpose of this paper is to develop a model to analyse the frequency response of a SAW device with FIDTs based on concentric wave surfaces. First of all, we employed a pair of FIDTs with the shape as the concentric wave surfaces to construct a focused SAW device. To calculate its frequency response, a simulation model was established by combining the 2D SAW filter model [3,4], effective permittivity approach [5,6] and perturbation theory [7-9]. Several designs of focused SAW devices are simulated and discussed. Further, we fabricated these devices using the 
(a)

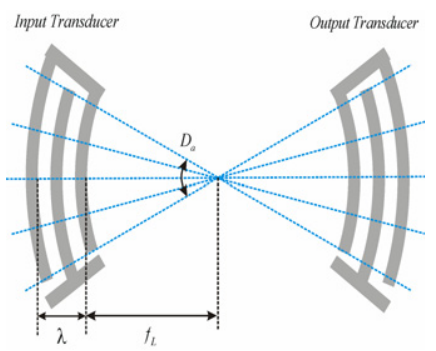

(b)

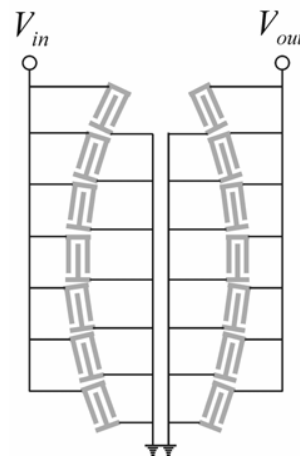

Figure 1. Illustrations of a focused SAW device. (a) Equivalent aperture separations of a focused SAW device. $(b)$ Parallel connection of segments of a focused SAW device.

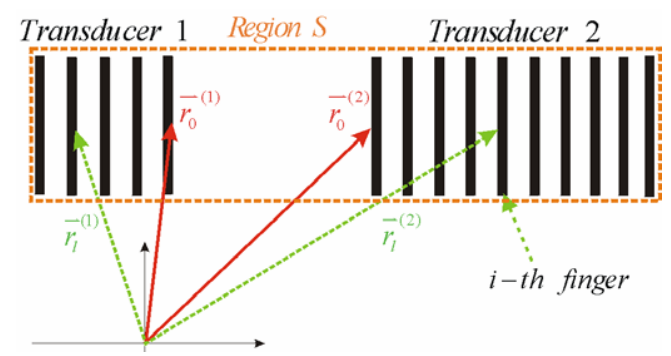

Figure 2. Geometry of a conventional SAW filter.

microelectromechanical system (MEMS) process technology. Finally, we discussed the features of the frequency responses according to the simulated and measured results.

\section{Simulation model based on angular spectrum}

Shown in figure 1(a) is the illustration of a focused SAW device which was constructed by a pair of FIDTs based on concentric wave surfaces. In this figure, $D_{\mathrm{a}}, f_{\mathrm{L}}$ and $\lambda$ represent degree of arcs, geometric focal length and wavelength of the FIDT, respectively. As shown in figure 1(b), we segmented the focused SAW device degree by degree, and every segment can be treated as an individual SAW filter with small aperture. To analyse a curved segmented IDT with the equi-phase distribution, we adopted the equivalent aperture method, proposed by Kharusi and Farnell [1], to approximate it to a straight IDT with an equi-phase distribution. In addition, since not all of the segmented SAW filters are set in the minimum diffraction orientations [10], the second order effects of wave propagation, such as diffraction effect, refraction effect and beam steering, cannot be neglected. The conventional 1D SAW filter models, like the equivalent circuit model, coupling of modes $(\mathrm{COM})$ model $[11,12] \ldots$, etc are not suitable to simulate the frequency response of a focused SAW device. Therefore, we combined the 2D SAW filter model, angular spectrum of plane wave theory (ASoW) [3, 4], and the equivalent aperture separations method to analyse the frequency response of a segmented FIDT that considers the second order effects.

A SAW filter with uniform IDTs is illustrated in figure 2 , and the transducer radiation admittance parameters

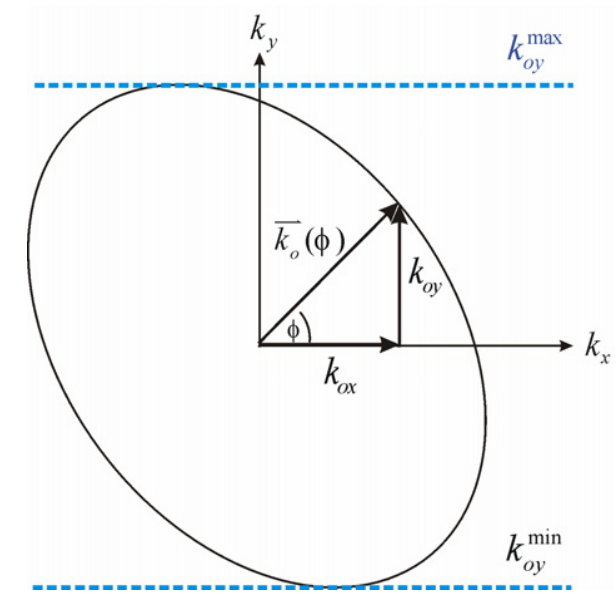

Figure 3. Slowness curve and the corresponding wave vectors.

of transducer 1 are expressed as [3,4]

$$
Y_{11}(\omega)=G^{(1)}(\omega)+j\left(\omega C_{\mathrm{s}}^{(1)}+B^{(1)}(\omega)\right),
$$

where $G^{(1)}(\omega)$ is the SAW radiation conductance and $B^{(1)}(\omega)$ is the SAW radiation susceptance. They are taken as

$$
G^{(1)}(\omega)=\omega L\left\{\left|\sum_{i=1}^{N^{(1)}} \bar{\rho}_{i}^{(1)}\left(\vec{k}^{(1)}\right) \mathrm{e}^{\mathrm{j} \vec{k} \cdot \vec{r}_{i}^{(1)}}\right|^{2}\right\}
$$

and

$$
\begin{aligned}
& B^{(1)}(\omega)=\omega L\left\{\sum_{i=1}^{N^{(1)}} \bar{\rho}_{i}^{(1)}\left(\vec{k}^{(1)}\right) \sum_{l=i+1}^{N^{(1)}} \bar{\rho}_{l}^{(1)}\left(\vec{k}^{(1)}\right)\right. \\
& \left.\times \sin \left[\vec{k}^{(1)} \cdot\left(\vec{r}_{i}^{(1)}-\vec{r}_{l}^{(1)}\right)\right]\right\},
\end{aligned}
$$

where $N^{(1)}$ is the number of finger of transducer 1 and $C_{\mathrm{s}}^{(1)}$ is the static capacitance. The theoretical value of the static capacitance can be found in [13]. $\quad \bar{\rho}_{i}^{(1)}\left(\vec{k}^{(1)}\right)$ is the Fourier transform of the 2D charge distribution of $i$ th finger of transducer 1 and defined as

$$
\rho_{i}(x, y)=Q_{f}(x) \cdot\left[H\left(y_{i}+W\right)-H\left(y_{i}-W\right)\right],
$$

where $H$ represents Heaviside's step function, $Q_{f}(x)$ is the elementary charge density [14] and $W$ is the aperture of the SAW filter. The integral operators in equations (1)-(3), $L\{\cdots\}$, are defined by

$$
L\{\cdots\}=\frac{1}{2 \pi} \int_{k_{0 y}^{\min }}^{k_{0 y}^{\max }} \frac{\Gamma\left(k_{0 y}\right)}{\gamma\left(k_{0 y}\right)} \cdots \mathrm{d} k_{0 y},
$$

where $k_{0 y}=k_{0}(\phi) \sin \phi$ is the wave vector component of SAW along the $y$-axis. The maximal and minimal values of $k_{0 y}, k_{0 y}^{\max }$ and $k_{0 y}^{\min }$ (as shown in figure 3 [3]) depend on the slowness curve of SAW propagating in the substrate. $\phi$ is the angle between the propagation direction and the $x$-axis. The coupling factor $\Gamma\left(k_{0 y}\right)$ and a defined factor $\gamma\left(k_{0 y}\right)$ can be expressed as

$$
\Gamma\left(k_{0 y}\right)=\frac{1}{\varepsilon_{\text {eff }}^{\infty}\left(k_{0 y}\right)} \frac{k_{0}^{2}\left(k_{0 y}\right)-k_{\infty}^{2}\left(k_{0 y}\right)}{2 k_{0}^{2}\left(k_{0 y}\right)}
$$


and

$$
\frac{1}{\gamma\left(k_{0 y}\right)}=\frac{1}{k_{0}^{2}\left(k_{0 y}\right)}\left(k_{0 x}\left(k_{0 y}\right)-k_{0 y} \frac{\mathrm{d} k_{0 x}\left(k_{0 y}\right)}{\mathrm{d} k_{0 y}}\right),
$$

where $\varepsilon_{\text {eff }}^{\infty}\left(k_{0 y}\right)$ is the effective permittivity as a function of $k_{0 y}$ when $\omega \rightarrow \infty$. $k_{\infty}\left(k_{0 y}\right)$ is the magnitude of SAW vector on the metalized surface (short circuit condition). In this study, the effective permittivity and Rayleigh wave velocity are calculated using the effective permittivity approach. The reason why we chose the effective permittivity approach to calculate the Rayleigh wave velocity is that this formulation can be easily extended to analyse the case of a layered SAW device [5,6]. Similarly, the transducer radiation admittance of transducer 2 can be determined by replacing superscript 1 in equations (1)-(3) by superscript 2.

Moreover, the transmission admittance parameter for the two transducers can be expressed as $[3,4]$

$Y_{12}(\omega)=\omega L\left\{\bar{\rho}^{(2)}\left(-\vec{k}^{(2)}\right) \cdot \bar{\tau}^{(f)}\left(\vec{k}^{(f)}\right) \cdot \bar{\rho}^{(1)}\left(\vec{k}^{(1)}\right)\right\}$,

where $\bar{\tau}^{(f)}\left(\vec{k}^{(f)}\right)$ represents the wave propagation on the free surface between the two transducers. It is taken as

$$
\bar{\tau}^{(f)}\left(\vec{k}^{(f)}\right)=\exp \left[-j \vec{k}^{(f)} \cdot\left(\vec{r}_{0}^{(2)}-\vec{r}_{0}^{(1)}\right)\right],
$$

where $\vec{k}^{(f)}$ is the Rayleigh wave vectors on the free surface (open circuit condition). $\vec{k}^{(1)}$ and $\vec{k}^{(2)}$ mean the perturbed Rayleigh wave vectors in the transducer areas and can be computed by [7-9]

$$
\vec{k}^{t}(\phi)=\frac{\omega}{v_{\mathrm{R}}^{\prime}(\phi)}, \quad t=1,2
$$

and

$v_{\mathrm{R}}^{\prime}=v_{\mathrm{R}}\left(1+\frac{\Delta v_{\mathrm{R}}}{v_{\mathrm{R}}}\right)=v_{\mathrm{R}}\left[1+D_{k}\left(\frac{K_{\mathrm{e}}^{2}}{2}\right)+D_{m}\left(\frac{h}{\lambda}\right)\right]$,

where $v_{\mathrm{R}}$ is the free-surface Rayleigh wave velocity and $v_{\mathrm{R}}^{\prime}$ is the perturbed Rayleigh wave velocity in the transducer areas. $\Delta v_{\mathrm{R}}$ is the difference between $v_{\mathrm{R}}^{\prime}$ and $v_{\mathrm{R}} . K_{\mathrm{e}}^{2}$ is the electromechanical coefficient, $h$ is the thickness of the thin film electrodes and $\lambda$ is the wavelength of the generated SAW. The functions of $D_{k}$ and $D_{m}$ are expressed as

$$
D_{k}=-\frac{1}{2}\left[1+\frac{P_{s}(-\cos (\pi \eta))}{P_{s-1}(-\cos (\pi \eta))}\right]
$$

and

$$
\begin{aligned}
D_{m}= & \frac{\eta \pi K_{\mathrm{e}}^{2}}{\varepsilon_{\mathrm{eff}}^{\infty}}\left[\left|\frac{U_{x}}{\varphi}\right|^{2}\left(\alpha_{1}-\rho^{\prime} v_{\mathrm{R}}^{2}\right)+\left|\frac{U_{y}}{\varphi}\right|^{2}\left(\alpha_{2}-\rho^{\prime} v_{\mathrm{R}}^{2}\right)\right. \\
& \left.-\left|\frac{U_{z}}{\varphi}\right|^{2} \rho^{\prime} v_{\mathrm{R}}^{2}\right],
\end{aligned}
$$

where $U_{x}, U_{y}$ and $U_{z}$ are the particle displacements along the $x$-, $y$ - and $z$-axis, respectively. $P_{v}(\xi)$ is a Legendre function of degree $v$ with a variable $\xi$. $\varphi$ is the surface electrical potential and $\eta$ is the metallization ratio of IDT. $\rho^{\prime}, \lambda^{\prime}$ and $\mu^{\prime}$ are the density and Lame constants of the electrode material. In addition,

$$
\alpha_{1}=\frac{4 \mu^{\prime}\left(\lambda^{\prime}+\mu^{\prime}\right)}{\lambda^{\prime}+2 \mu^{\prime}}
$$

and

$$
\alpha_{2}=\mu^{\prime}
$$

Similarly, the transmission admittance $Y_{21}(\omega)$ also can be evaluated by interchanging superscript 1 and superscript 2 in equations (8) and (9). We note that in order to consider the refraction effect accurately, the $k_{y}$ components of $\vec{k}^{(1)}, \vec{k}^{(2)}$ and $\vec{k}^{(f)}$ must be equal to $\vec{k}_{0 y}$.

\section{Frequency response of a focused SAW device}

Through the above derivations, four elements of the admittance matrix of a conventional SAW filter, $Y_{11}, Y_{12}, Y_{21}$ and $Y_{22}$, can be determined. That is to say, the admittance matrix of every segmented SAW device can be determined by using the formulation. In this section, we will extend it to simulate the frequency response of a focused SAW device. As shown in figure $1(b)$, every segmented input and output transducer has the same input and output voltage $\left(V_{\text {in }}\right.$ and $\left.V_{\text {out }}\right)$, respectively. Thus, the equivalent circuit of a focused SAW device can be viewed as a parallel circuit of all segmented SAW devices, and

$$
\begin{gathered}
V_{\text {in }}^{i}=V_{\text {in }}, \\
V_{\text {out }}^{i}=V_{\text {out }}, \\
I_{\text {in }}=I_{\text {in }}^{1}+I_{\text {in }}^{2}+\cdots+I_{\text {in }}^{n}=\sum_{i=1}^{n} I_{\text {in }}^{i}
\end{gathered}
$$

and

$$
I_{\mathrm{out}}=I_{\mathrm{out}}^{1}+I_{\mathrm{out}}^{2}+\cdots+I_{\mathrm{out}}^{n}=\sum_{i=1}^{n} I_{\mathrm{out}}^{i},
$$

where, $V_{\text {in }}^{i}$ and $V_{\text {out }}^{i}$ are the voltages of the $i$ th segment of the input and output transducers, respectively. $I_{\text {in }}^{i}$ and $I_{\text {out }}^{i}$ are the bus bar currents of the $i$ th segment of the input and output transducer, respectively. $I_{\text {in }}$ and $I_{\text {out }}$ are the bus bar currents of the input and output whole focused transducers, respectively. Since the focused SAW device is a two-port SAW device, the relation between its voltages, currents and total admittance matrix can be taken as

$$
\left\{\begin{array}{c}
I_{\text {in }} \\
I_{\text {out }}
\end{array}\right\}=\left[\begin{array}{ll}
Y_{11} & Y_{12} \\
Y_{21} & Y_{22}
\end{array}\right]\left\{\begin{array}{c}
V_{\text {in }} \\
V_{\text {out }}
\end{array}\right\} .
$$

Every element of the total admittance parameters is computed by

$$
\begin{array}{ll}
Y_{11}=\sum_{i=1}^{n}\left[Y_{11}^{i}\right] & Y_{12}=\sum_{i=1}^{n}\left[Y_{12}^{i}\right], \\
Y_{21}=\sum_{i=1}^{n}\left[Y_{21}^{i}\right] & Y_{22}=\sum_{i=1}^{n}\left[Y_{22}^{i}\right],
\end{array}
$$

where $Y_{12}^{i}$ and $Y_{21}^{i}$ are the transmission admittance parameters of the $i$ th segment of a focused SAW device. $Y_{11}^{i}$ and $Y_{22}^{i}$ are the transducer radiation admittance parameters of the $i$ th segment of a focused SAW device.

Since the $i$ th segmented output transducer is set in the direction of energy velocity of the $i$ th segmented input transducer, their apertures are parallel and the equivalent aperture separations method is still valid. The illustration is 

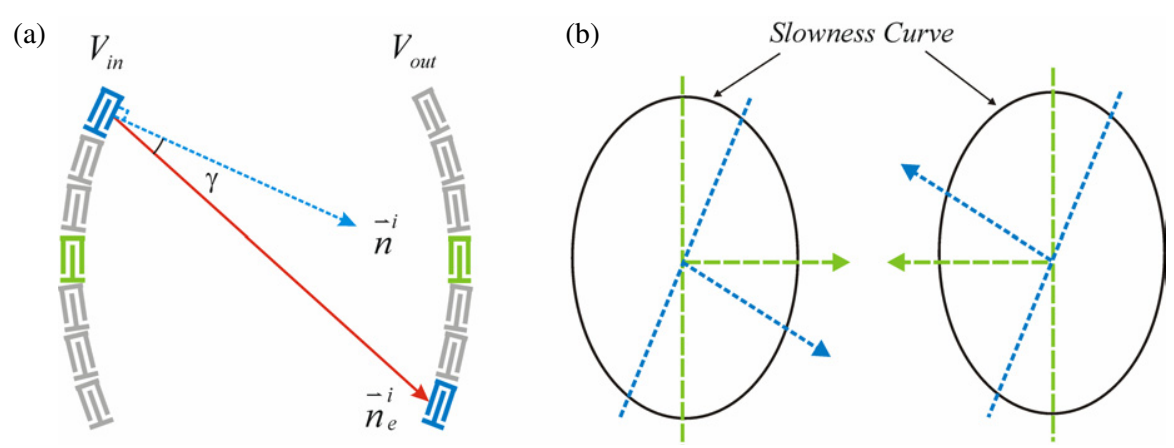

Figure 4. Segments of a focused SAW device with their individual orientations.

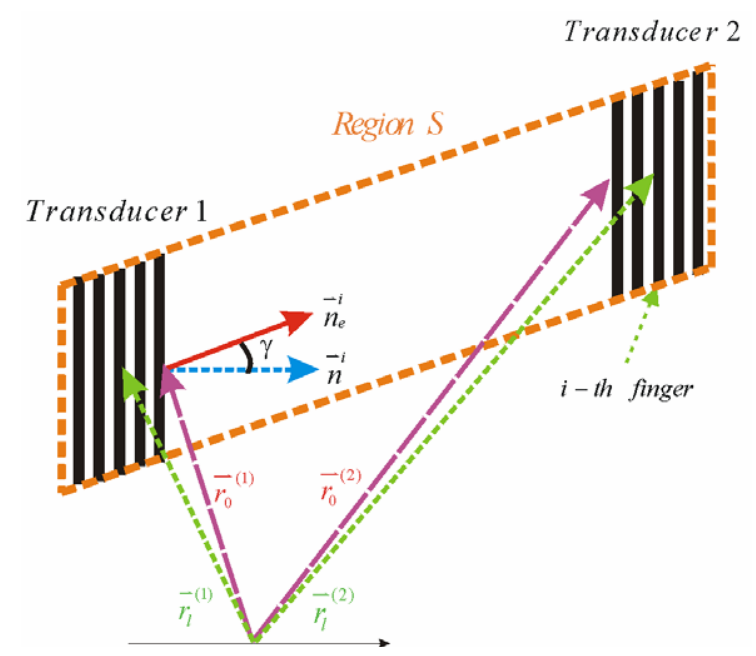

Figure 5. Geometry of the $i$ th segment of a focused SAW device.

shown in figure $4(a)$, where $\vec{n}^{i}, \vec{n}_{\mathrm{e}}^{i}$ and $\gamma$ are the phase velocity direction, the energy velocity direction and the beam steering angle of the $i$ th segment, respectively. The relative geometry of the $i$ th segment is shown in figure 5. Since the orientations of all segments are different, the integration operator $L_{i}\{\cdots\}$ of the $i$ th segment of the focused SAW device must be modified as

$$
L_{i}\{\cdots\}=\frac{1}{2 \pi} \int_{\left(k_{0 y}^{\min }\right)_{i}}^{\left(k_{0 y}^{\max }\right)_{i}} \frac{\Gamma\left(k_{0 y}\right)}{\gamma\left(k_{0 y}\right)} \cdots \mathrm{d} k_{0 y},
$$

where $\left[\left(k_{0 y}^{\min }\right)_{i},\left(k_{0 y}^{\max }\right)_{i}\right]$ is the integration interval of the $i$ th segment and depends on the slowness curve (shown in figure $4(b)$ ) and the orientation of the $i$ th segment. We note that for the $i$ th segment, the input and output transducers must be set on different sides of the slowness curve. This point is very important for calculating the admittance parameters of the segments of a focused SAW device. By substituting the admittance matrixes of all segments into equation (21), the admittance matrix of a focused SAW device can be determined. The scattering parameter $S_{21}$ of the focused SAW device can be obtained from [4]

$$
S_{21}=\frac{-2 \cdot Y_{21} \sqrt{Y_{1} Y_{2}}}{\left(Y_{11}+Y_{1}\right)\left(Y_{22}+Y_{2}\right)-Y_{21} Y_{12}},
$$

where $Y_{1}$ and $Y_{2}$ are the source (transducer 1) and load (transducer 2) admittances, respectively. In general, since the
Table 1. Design parameters of different focused SAW transducers

\begin{tabular}{lclll}
\hline $\begin{array}{l}\text { Design } \\
\text { parameters }\end{array}$ & $\begin{array}{l}\text { Number } \\
\text { of pairs } \\
\left(N_{\mathrm{p}}\right)\end{array}$ & $\begin{array}{l}\text { Degree } \\
\text { of arcs } \\
\left(D_{\mathrm{a}}\right)\end{array}$ & $\begin{array}{l}\text { Geometric } \\
\text { focal length } \\
\left(f_{\mathrm{L}}\right), \lambda\end{array}$ & $\begin{array}{l}\text { Wavelength } \\
(\lambda), \\
\mu \mathrm{m}\end{array}$ \\
\hline Design 1-1 & 5 & $40^{\circ}$ & 50 & 58.13 \\
Design 1-2 & 10 & $40^{\circ}$ & 50 & 58.13 \\
Design 1-3 & 20 & $40^{\circ}$ & 50 & 58.13 \\
Design 2-1 & 10 & $20^{\circ}$ & 50 & 58.13 \\
Design 2-2 & 10 & $40^{\circ}$ & 50 & 58.13 \\
Design 2-3 & 10 & $60^{\circ}$ & 50 & 58.13 \\
\hline
\end{tabular}

source and load admittances are equal to $50 \Omega$, the frequency response of a focused SAW device, insertion loss (IL), can be taken as

$$
\mathrm{IL}=20 \log \left|S_{21}\right|(\mathrm{dB}) .
$$

In the following section, we simulate the frequency responses of focused SAW devices with different designs based on the model presented above. The design parameters used in the calculations are shown in table 1. In addition, the thickness and metallization ratio of the metal electrode are $160 \mathrm{~nm}$ and $0.5 \mathrm{~nm}$, respectively. First of all, we analysed the wave characteristics of the $\mathrm{YZ}-\mathrm{LiNbO}_{3}$ substrate based on the effective permittivity approach. Figures $6(a),(b)$ and $(c)$ are the plots of Rayleigh wave velocity, effective permittivity and electromechanical coefficient as a function of propagation direction, respectively. From the three figures, we find that the anisotropy of $\mathrm{YZ}-\mathrm{LiNbO}_{3}$ is obvious. Figure 7(a) shows the simulated frequency responses corresponding to different number of pairs (Design 1-1 to 1-3 of table 1). In figure 7(a), the pass-band width becomes narrower and the insertion loss becomes smaller with the increase in the number of pairs. However, once the number of pairs is large enough, the insertion loss will not decrease with the increase in the number of pairs anymore. But, the scale of the device will increase. Hence, the number of pairs of a focused SAW device must be optimized according to the desired pass-band width and insertion loss. Figure $7(b)$ shows the simulated frequency responses corresponding to different degree of arcs (Design $2-1$ to $2-3$ of table 1 ). From the results of Design $2-1$ and $2-2$ shown in this figure, we can see that when the degree of arcs of an FIDT is increased, the pass-band width and insertion loss do not change obviously. But, further comparing the results of Design 2-1 and 2-2 with those of Design 2-3, we find that if the degree of arcs is too large, the insertion loss becomes larger 

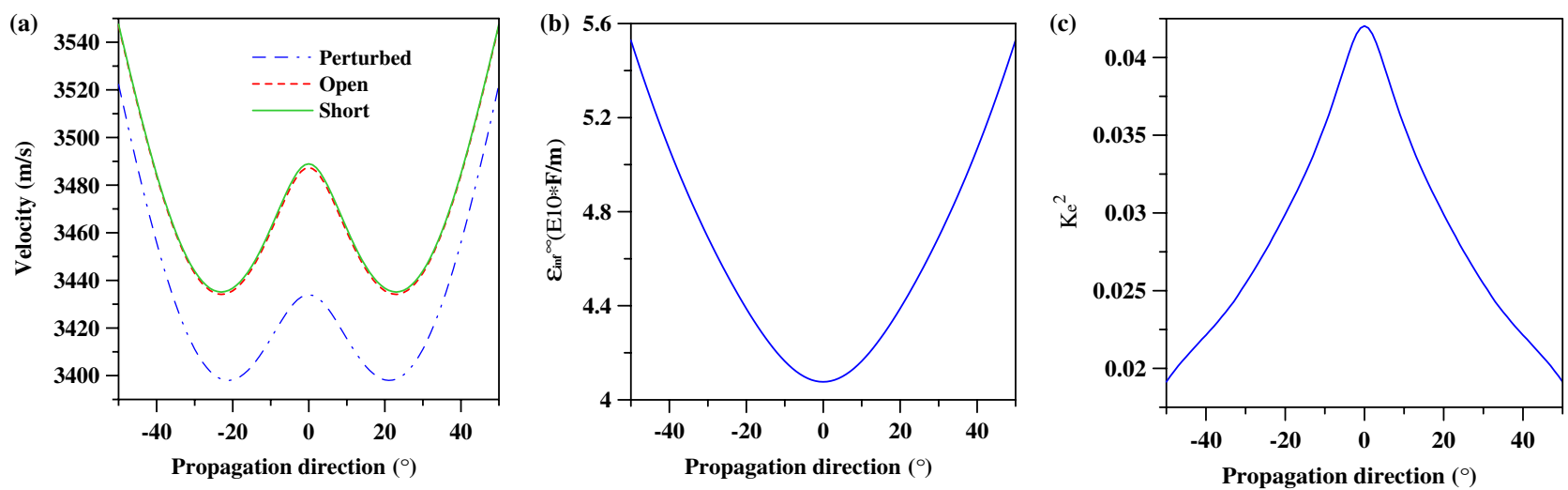

Figure 6. Parameters used in the calculation: (a) Rayleigh wave velocity. (b) Effective permittivity when $\omega \rightarrow \infty$. (c) Electromechanical coefficient.
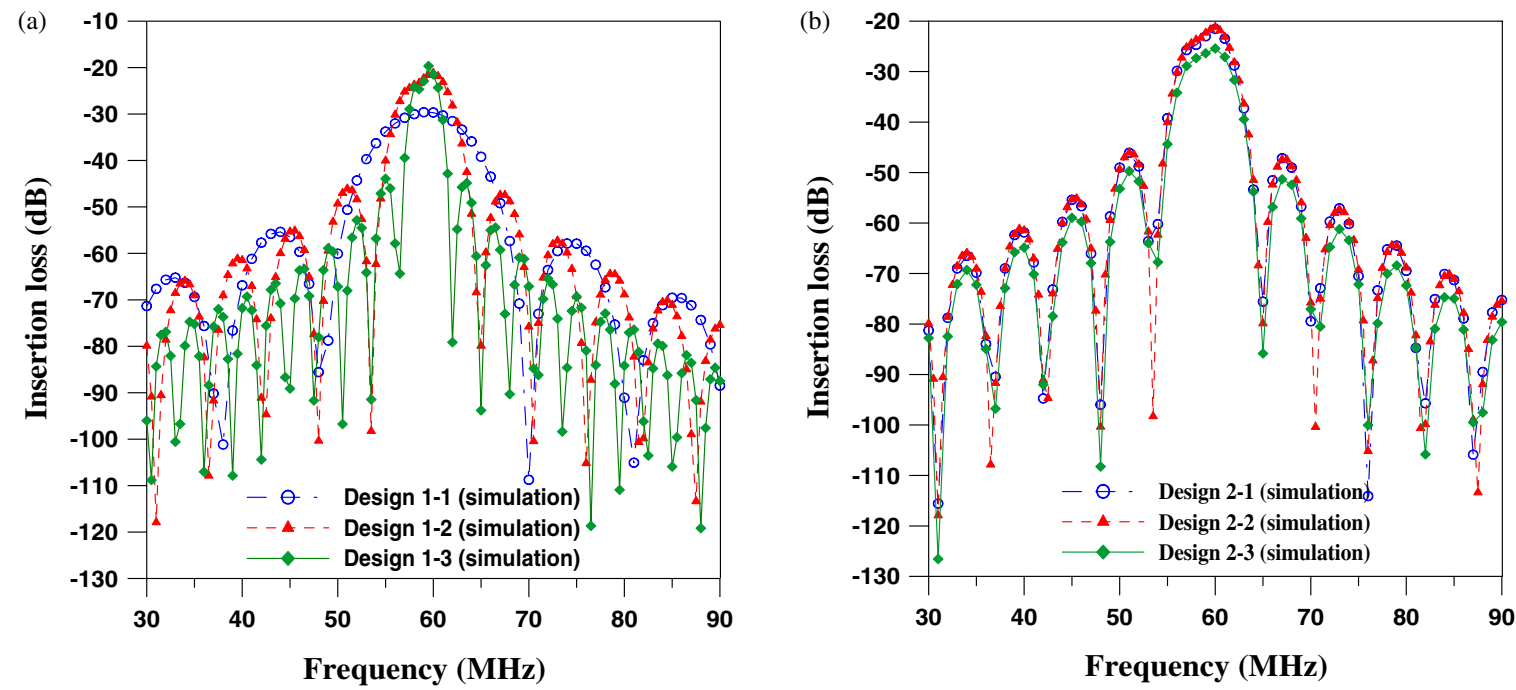

Figure 7. Simulated frequency responses of focused SAW devices of (a) Design 1-1 through 1-3 and (b) Design 2-1 through 2-3.
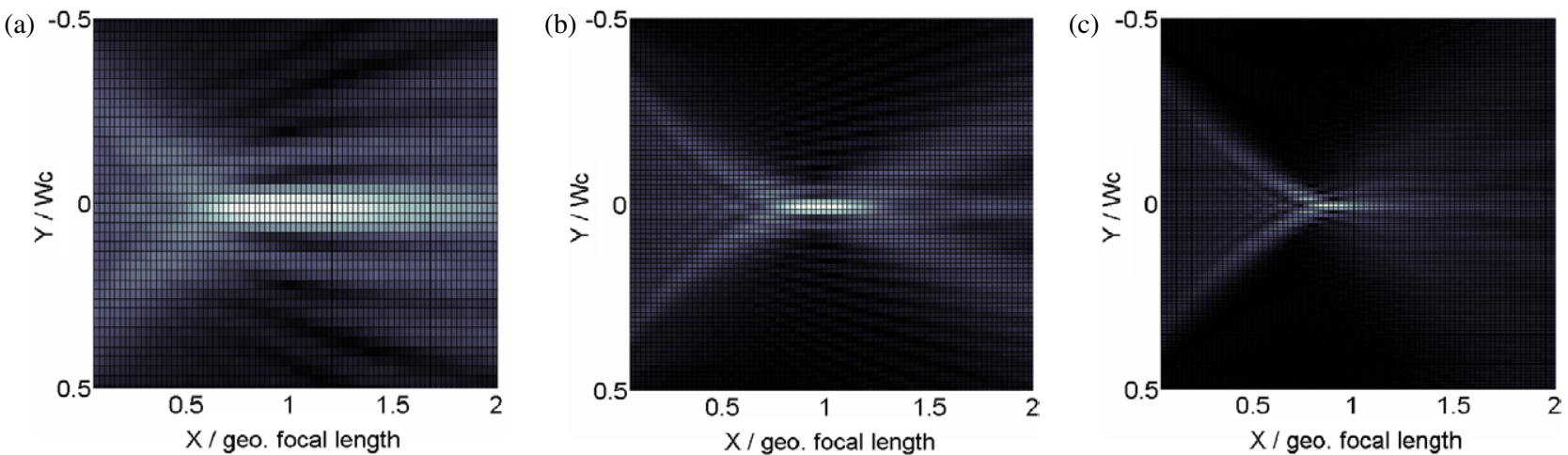

Figure 8. Simulated amplitude fields of focused SAW devices of (a) Design 2-1, (b) Design 2-2 and (c) Design 2-3. (The units of the horizontal and vertical axes are normalized geometric focal lengths and normalized apertures.)

obviously. On the other hand, as shown in figure 8 , when we increase the degree of arcs of an FIDT shaped as the concentric wave surfaces, the focusing property becomes better and the amplitude field approaches a focal point. The FIDT with the larger degree of arcs (Design 2-3) produces a smaller focal spot, but in the meantime, the frequency response of Design 2-3 has a larger insertion loss than that of Design 2-2 due to the diffraction effect. In other words, it is hard to design a focused SAW device with better focusing properties and lower insertion loss at the same time. There must be a compromise between better focusing properties and lower insertion loss. Therefore, we suggest that the FIDT with the larger degree of arc is suitable for use as an actuator or exciter due to its better focusing properties, while the one with the smaller degree of 
arc is more qualified to be a sensor or filter due to its smaller insertion loss.

\section{Fabrication and measurement of a focused SAW device}

In this section, we describe the fabrication process of the focused SAW device adopted in this study. The fabrication process can be simply described as follows:

(I) Cleaning. Put the piezoelectric wafer (e.g. $\mathrm{YZ}-\mathrm{LiNbO}_{3}$ ) into a beaker which is filled with acetone solution. Then, place the beaker onto an ultrasonic cleaner to vibrate and clean the wafer for about $5 \mathrm{~min}$. Next, clip the wafer and put it into another beaker which is filled with methyl alcohol solution for about $5 \mathrm{~min}$ to clean the residual acetone. And finally, use deionized water (DI water) to rinse the wafer and the cleaning process is complete.

(II) Metallization. After the cleaning process, use the thermal evaporator machine to evaporate the aluminium thin film onto the wafer.

(III) Photoresist (PR) coating. In general, PR is used as the etching mask in order to let the aluminium locate on the desired area. Therefore, use Spin Coater to coat the PR (S1813) onto the wafer uniformly and then put the wafer on a hot plate and soft bake it at $90^{\circ} \mathrm{C}$ for about $75 \mathrm{~s}$.

(IV) Exposure and development. Further, put the spun PR into a Mask Aligner and expose it for about $14 \mathrm{~s}$ in order to transfer the patterns of photo mask to the spun PR. After the exposure process, dip the wafer into the develop solution (MF319) for about $45 \mathrm{~s}$ to develop the patterns of a focused SAW device. Finally, put the wafer on a hot plate and hard bake it at $90^{\circ} \mathrm{C}$ for about $20 \mathrm{~min}$.

$(V)$ Etching. Next, soak the wafer with etching mask (PR) into a beaker which is filled with aluminium etching solution $(80 \%$ $\mathrm{H}_{3} \mathrm{PO}_{4}, 5 \% \mathrm{HNO}_{3}, 5 \% \mathrm{CH}_{3} \mathrm{COOH}$ and $\left.10 \% \mathrm{H}_{2} \mathrm{O}\right)$ to etch the undesired area of the aluminium thin film for about $2 \mathrm{~min}$. The etching time depends on the thickness of aluminium and must be controlled carefully. If the etching time is too long, the aluminium will be undercut and the shape of the patterns will be no longer what we want. After the etching process, put the wafer into DI water to clean it.

(VI) $P R$ removing. Put the wafer into a beaker which is filled with acetone solution and then place the beaker onto an ultrasonic cleaner for about $5 \mathrm{~min}$ to remove the PR. In order to clean the residual acetone, put the wafer into a beaker which is filled with methyl alcohol solution for about $5 \mathrm{~min}$. After cleaning, dip the wafer into DI water to rinse the wafer again. Finally, put the wafer on a hot plate and bake it at $120^{\circ} \mathrm{C}$ for about $10 \mathrm{~min}$ to remove the residual moisture.

Through these fabrication processes, a focused SAW device can be realized. The finished products of focused SAW devices are shown in figure 9. The focused SAW devices are fabricated on 4 inch $\mathrm{YZ}-\mathrm{LiNbO}_{3}$ wafers. The back side roughness of the wafers are around $1.21-1.54 \mu \mathrm{m}$ in order to decrease the effect of bulk acoustic wave (BAW). After fabrication, we used a network analyzer (Agilent 8714ES RF Network Analyzer) to measure the frequency responses of the focused SAW devices. Figure 10 shows the experimental results of Design 1-2 in table 1. In figure 10(a), the ripples of pass-band are about $\pm 0.4 \mathrm{~dB}$, the $3 \mathrm{~dB}$ band width is about $4 \mathrm{MHz}$ and the stop-band rejection is about $25 \mathrm{~dB}$. In addition, we find that when the frequency is higher than $80 \mathrm{MHz}$, a high frequency signal appears. It may be the BAW signal or the electromagnetic feedthrough effect. In order to figure out this problem, we transformed the frequency domain signal into the time domain and the result is shown in figure $10(b)$. In this figure, there is a high-intensity signal at $0.1-0.8 \mu \mathrm{s}$. Because the BAW velocity of $\mathrm{YZ}-\mathrm{LiNbO}_{3}$ is about $7220 \mathrm{~m} \mathrm{~s}^{-1}$, it takes more than $0.5 \mu$ s to arrive at the output transducer. Therefore, the initial signal shall be the electromagnetic signal. In order to obtain the frequency response without considering the electromagnetic feedthrough effect, we picked the time domain signal after $0.5 \mu \mathrm{s}$ and inversely transformed it into frequency domain. The result is shown in figure 11(a). We find that the high frequency signal is eliminated obviously. Thus, we conclude that the high frequency signal is indeed electromagnetic feedthrough effect and not BAW signal. Even though we removed the electromagnetic signal, the ripples of pass-band still exist. In figure 10(b), two ripples can be found at about 1.9 and $5.5 \mu \mathrm{s}$. They are the direct SAW response and the triple transit response (TTR), respectively. In order to obtain the frequency response of the direct SAW, we only picked the time-domain signal between 1.6 and $2.5 \mu \mathrm{s}$ and inversely transformed it into frequency domain. The result is shown in figure $11(b)$. We find that the ripples of passband almost disappear and the pass-band becomes smoother. Therefore, the TTR is the major cause of the ripples of passband. The above signal processing is called 'time gating'. By using the process of time gating, the frequency response of the direct SAW, as shown in figure 11(b), can be obtained.

Overall, the frequency response of a focused SAW device based on concentric wave surfaces has the following features: (i) less ripples of pass-band and (ii) high stop-band rejection. These features are quite similar to the conventional two-port SAW devices with uniform IDTs. Therefore, the focused SAW devices are also suitable to be applied as sensors, detectors, actuators, filters etc. However, the amplitude field of this kind of focused SAW device is close to a focal point. This makes it more sensitive to variations in the focal area, instead of the whole delayline region. Therefore, the focused SAW device is quite suitable to excite or detect the variation within a very small area.

\section{Results and discussions}

First, we compare the experimental results with of different designs. Shown in figure $12(a)$ are the experimental results of Design 1-1 to $1-3$ of table 1 . Figure 12(a) shows the variations of frequency responses when we increase the number of pairs. In comparison with figure $7(a)$, good agreements can be found. Similarly, the experimental results of Design 2-1 to 2-3 in table 1 are shown in figure $11(b)$. The figure discusses the frequency responses of focused SAW devices with different degree of arcs. In comparison with figure $7(b)$, we find that the experimental results are quite consistent with the simulated ones.

Further, we compare the measured results with the simulated ones. Figures 13(a) and (b) show the experimental 
(a)

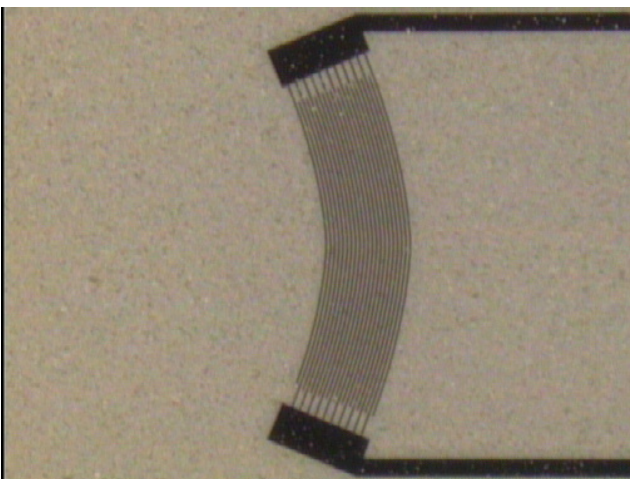

(b)

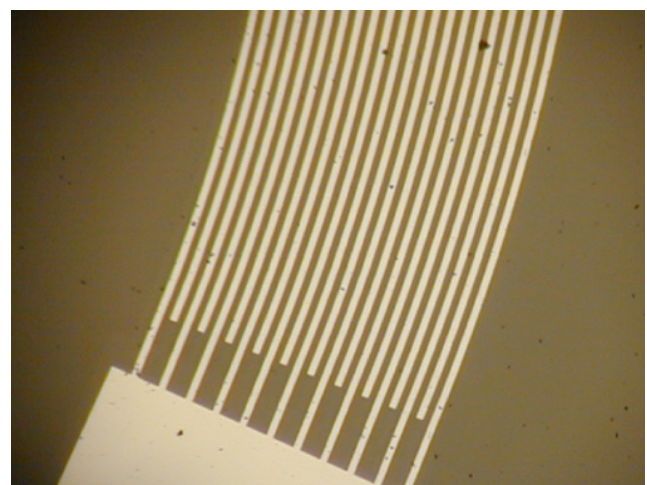

Figure 9. Images of the fabricated FIDT of Design 1-2 observed by a microscope of $(a) 15 \times$ and $(b) 50 \times$.
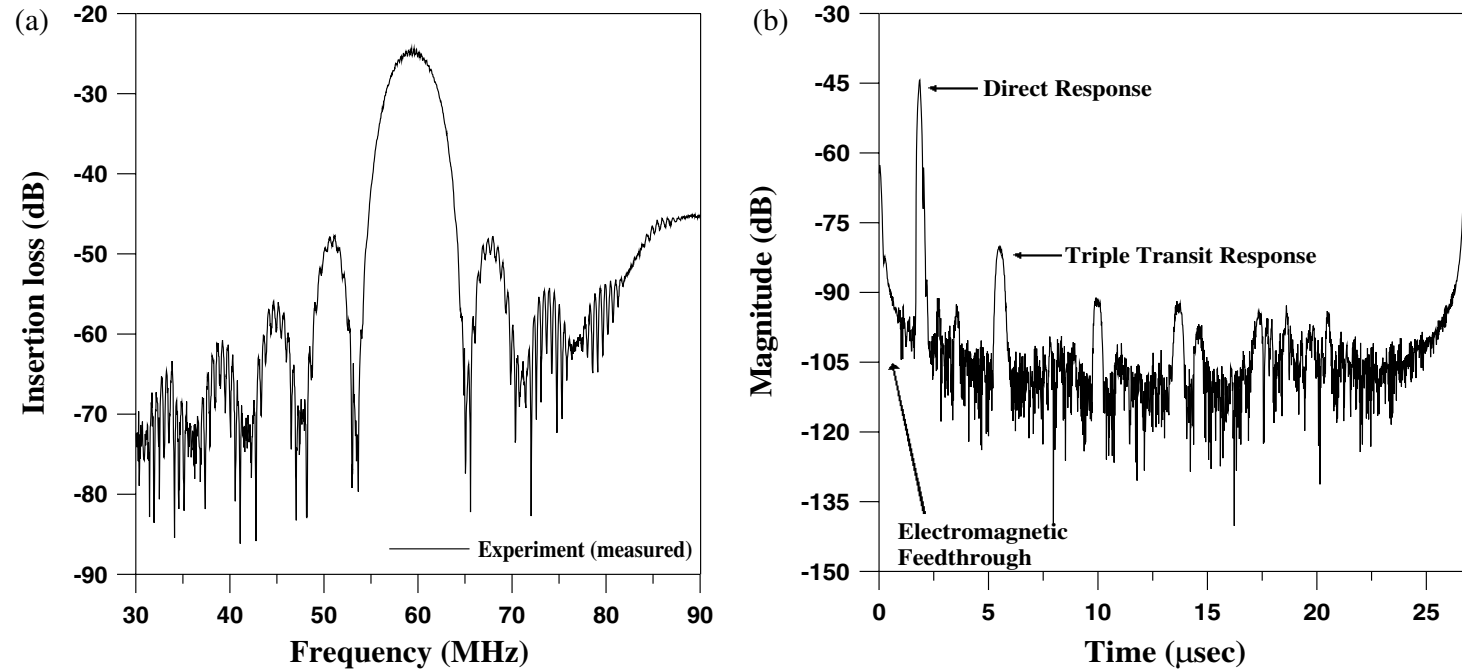

Figure 10. Experimental results of Design 1-2: $(a)$ the measured frequency-domain signal; $(b)$ the corresponding time-domain signal.
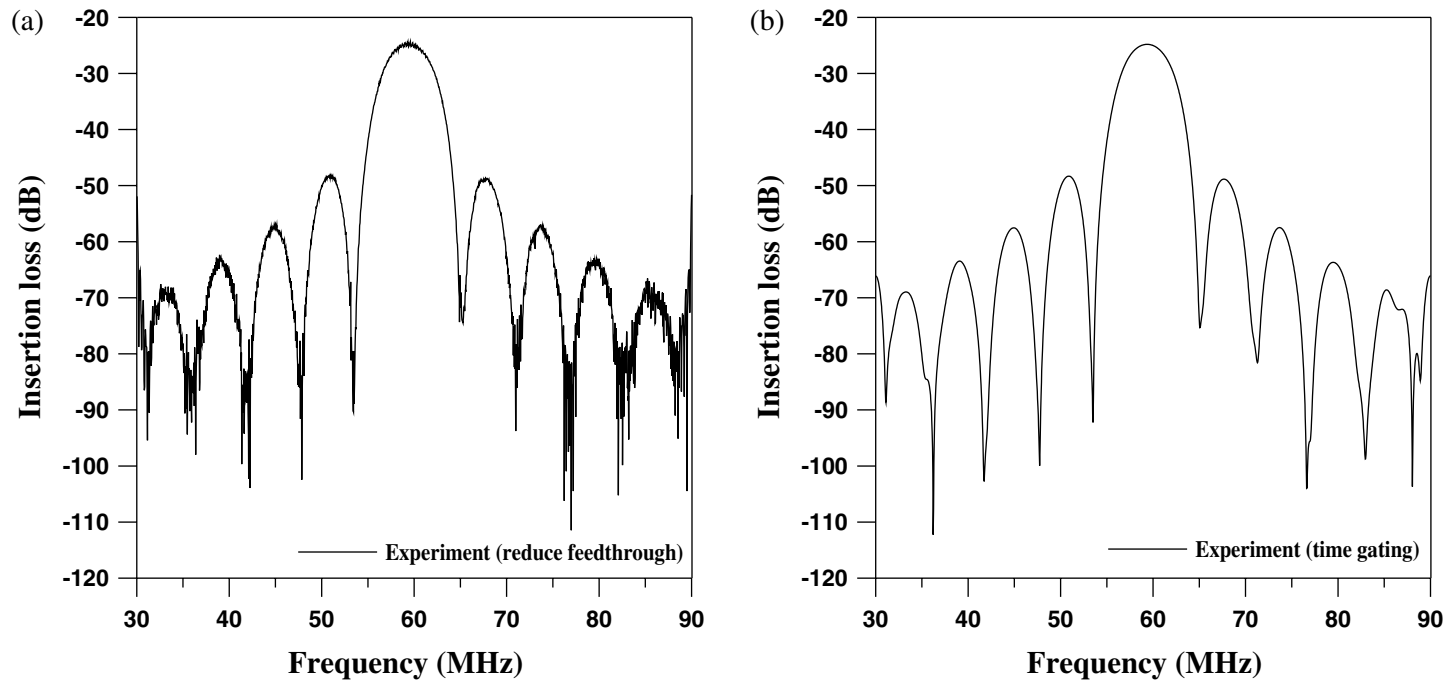

Figure 11. Experimental frequency responses of Design 1-2 (a) without electromagnetic feedthrough and $(b)$ without both electromagnetic feedthrough and triple transit response.

and simulated results of Design 1-1 and 1-2 in table 1, respectively. The simulated results agree well with the experimental results, but the insertion losses of experimental results are somewhat smaller than the simulated results. The larger insertion losses probably result from the following two reasons:

(I) Material properties. When we use the effective permittivity approach to determine the parameters, such as SAW velocity, electromechanical coupling coefficient, etc the material constants of the substrate are referred to in the 

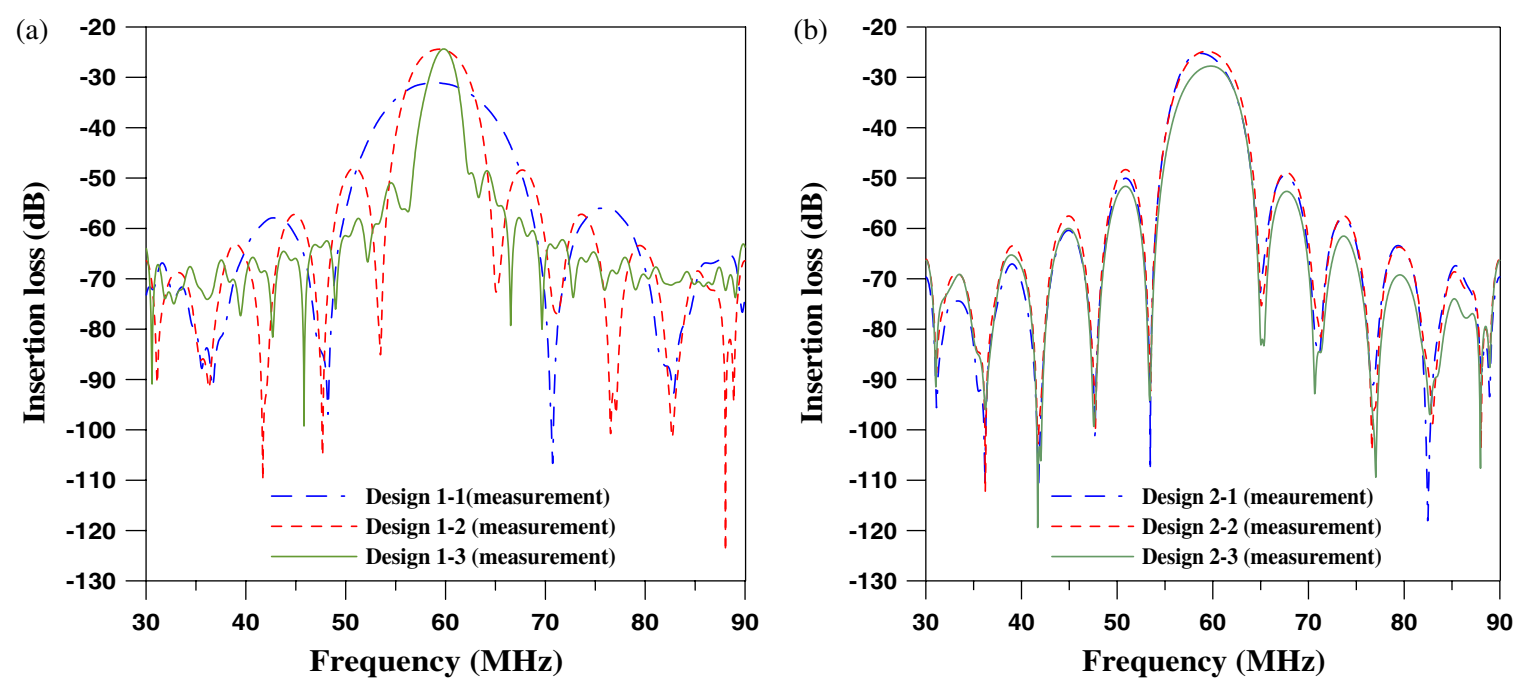

Figure 12. Experimental frequency responses of the direct SAW for (a) Design 1-1 through 1-3 and (b) Design 2-1 through 2-3.
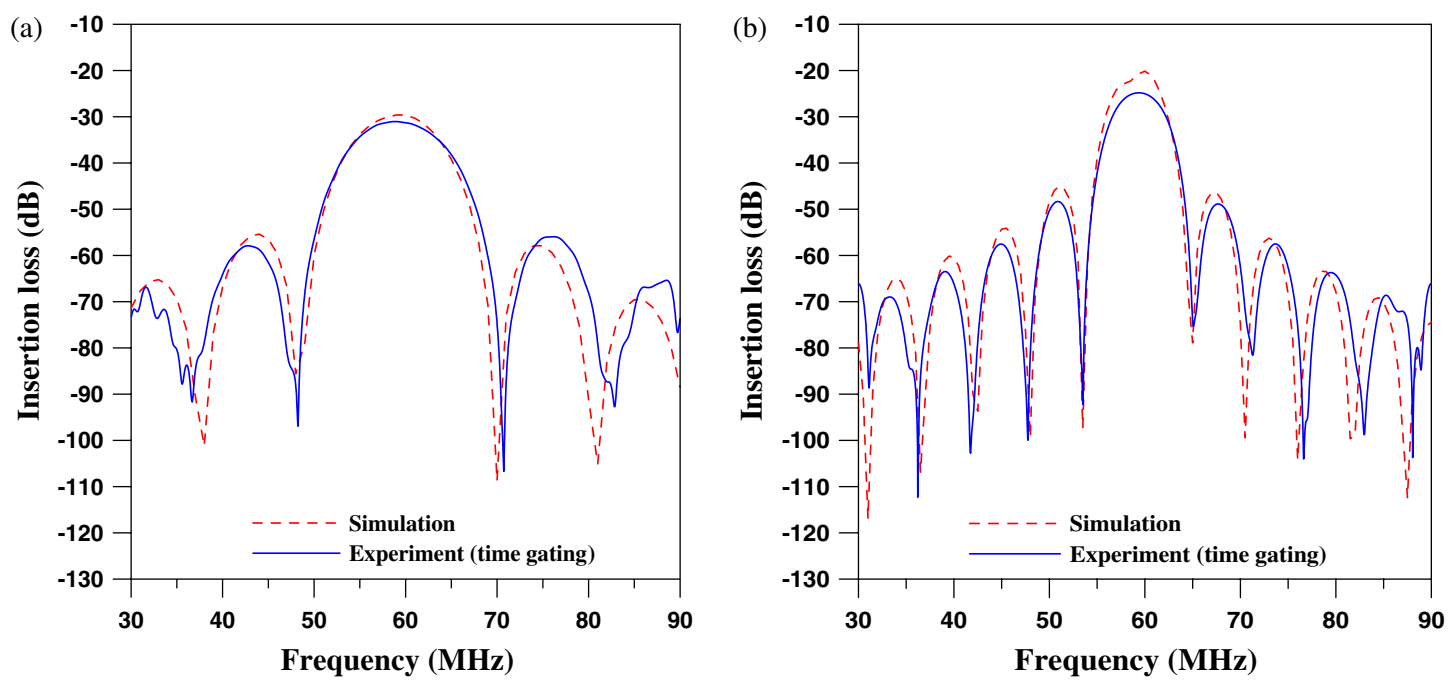

Figure 13. Comparisons between experimental and simulated results for $(a)$ Design 1-1 and (b) Design 1-2.

literature. But actually, the practical material constants may be somewhat different from the theoretical ones.

(II) Propagation loss. The simulation model presented in section 2 neglects the propagation loss. In fact, propagation loss is not equal to zero. It depends on the SAW frequency and the wave propagation distance. In order to meet the practical situation, adding the term of propagation loss into the function of wave propagation, $\bar{\tau}^{(f)}\left(\vec{k}^{(f)}\right)$, is needed (equation (9)). This awaits further study.

\section{Conclusions}

The frequency response of a focused SAW device based on concentric wave surfaces is first analysed. A simulation model for calculating the frequency response of a focused SAW device has been established by combining the 2D SAW filter model, the effective permittivity approach and the perturbation theory. We used the MEMS process technique to fabricate the focused SAW devices, and the experimental results are quite consistent with the simulated results. The frequency response of the focused SAW device has a few ripples of pass-band and high stop-band rejection. These features are very similar to conventional two-port SAW devices. Therefore, focused SAW devices are also suitable for the applications of conventional SAW devices, such as sensors, detectors, actuators, filters, etc. In addition, since their amplitude fields are close to a focal point, the focused SAW devices are suitable to excite or detect the variations within a very small area. In summary, we have developed a simulation model based on ASoW to calculate the frequency response of a focused SAW device, and verified it with experimental results. Some special features of the frequency response of a focused SAW device have been found and they may be useful in future applications.

\section{Acknowledgment}

The authors gratefully acknowledge the financial support of this research by the National Science Council of R.O.C. through the grant NSC92-2212-E-002-001. 


\section{References}

[1] Kharusi M S and Farnell G W 1972 On diffraction and focusing in anisotropic crystals Proc. IEEE 60 945-56

[2] Marynowski T J 1982 Focusing transducer for SAW beamwidth compression on YZ lithium niobate Proc. IEEE Ultrasonics Symp. pp 160-5

[3] Visintini G, Baghai-Wadji A and Manner O 1992 Modular two-dimensional analysis of SAW filters-Part I. Theory IEEE Trans. Ultrason. Ferroelectr. Freq. Control. 39 61-72

[4] Visintini G, Baghai-Wadji A and Manner O 1992 Modular two-dimensional analysis of SAW filters-Part II. Analysis and compensation results IEEE Trans. Ultrason. Ferroelectr. Freq. Control. 39 73-81

[5] Wu T T and Chen Y Y 2002 Exact analysis of dispersive $\mathrm{SAW}$ devices on $\mathrm{ZnO} /$ diamond/Si layered structures IEEE Trans. Ultrason. Ferroelectr. Freq. Control. 49 142-9

[6] Chen Y Y, Wu T T and Chou C T 2004 Analysis of the frequency response of a dispersive IDT/ZnO/sapphire
SAW filter using effectivity and coupling of modes model J. Phys. D: Appl. Phys. 37 120-7

[7] Datta S 1986 Surface Acoustic Wave Device (Englewood Cliffs, NJ: Prentice-Hall)

[8] Thorvaldsson T 1989 Analysis of the natural single phase unidirectional saw transducer Proc. IEEE Ultrasonics Symp. pp 91-6

[9] Thorvaldsson T and Abbott B P 1990 Low loss SAW filters utilizing the natural phase unidirectional transducer (NSPUDT) Proc. IEEE Ultrasonics Symp. pp 43-8

[10] Morgan D P 1985 Surface-Wave Devices for Signal Processing (Amsterdam: Elsevier) chapter 6

[11] Abbott B P 1989 A coupling-of-modes model for SAW transducers with arbitrary reflectivity weighting $P h D$ Dissertation University of Central Florida, Orlando

[12] Plessky V and Koskela J 2000 Coupling-of-modes analysis of SAW devices Int. J. High Speed Electron. Syst. 10 867-949

[13] Campbell C K 1998 Surface Acoustic Wave Devices for Mobile and Wireless Communications (San Diego: Academic)

[14] Morgan D P 1985 Surface-Wave Devices for Signal Processing (Amsterdam: Elsevier) appendix C 